\title{
TRATAMIENTO DE LA OBSTRUCCIÓN DEL CONDUCTO LACRIMAL CON EL STENT TEAR-LEADER
}

\author{
TREATMENT OF LACRIMAL DUCT OBSTRUCTION WITH \\ A TEAR-LEADER STENT
}

\author{
FERRER D ${ }^{1}$, ESTEBAN E $^{2}$, APARISI P ${ }^{2}$, COSÍN O ${ }^{2}$
}

\begin{abstract}
RESUMEN
Objetivo: Describir los resultados clínicos y estudiar la permeabilidad del stent de Tear-Leader $(\mathrm{PBN})$ en el tratamiento de la obstrucción del conducto lacrimal.

Método: Estudio prospectivo de 68 pacientes, remitidos desde el Servicio de Oftalmología para estudio de epífora por sospecha de obstrucción del conducto lacrimal. Hemos colocado un total de 74 stents Tear-Leader. Los criterios de inclusión fueron: obstrucción completa del conducto lacrimal con permeabilidad de canalículos y de puntos lacrimales así como ausencia de infección aguda. Hemos realizado en todos los pacientes el control clínico y una encuesta de satisfacción. Se realizó un estudio estadístico descriptivo y se evaluó la permeabilidad de la prótesis usando las curvas de Kaplan Meier.

Resultados: El seguimiento de los pacientes se realizó durante dos años. Se confirmó la resolución total de la epífora en el $77 \%$, (en el $23 \%$ persistió una epífora grado I). Permeabilidad del stent: la mediana fue de 490 días (15 meses) con rango de 11-730 días, al año la permeabilidad fue de 0,51 y a 2 años de 0,31. Refieren implantación dolorosa el $10 \%$ de los casos implantados. Aparecieron episodios de dacriocistitis leve en el $18 \%$ de los casos.

\footnotetext{
Recibido: 24/10/07. Aceptado: 28/10/09.

Hospital de la Rivera. Servicio de Radiología. Unidad de Radiología Vascular e Intervencionista. Valencia. España.

1 Doctora en Medicina y Cirugía.

${ }^{2}$ Licenciado en Medicina y Cirugía.

Correspondencia:

M. ${ }^{a}$ Dolores Ferrer Puchol

Unidad de Radiología Vascular Intervencionista

Servicio de Radiología

Hospital de la Ribera

Carretera de Corbera, km 1

46600 Alzira (Valencia)

España

E-mail: mferrer@hospital-ribera.com
}

\begin{abstract}
Purpose: To compare the effectiveness and patency of the TearLeader stent $(\mathrm{PBN})$ for treatment of lacrimal system obstruction.

Methods: A prospective study of 68 patients referred from the ophthalmology department with suspicion of lacrimal system obstruction. We placed 74 TearLeader PBN stents. Inclusion criteria were: complete obstruction of lacrimal duct with canalicula and lacrimal puncta patency, and absence of acute infection. We studied the clinical improvement by means of an opinion survey, and the patency of stents was evaluated with Kaplan-Meier survival curves.

Results: Follow-up of patients was two years. A painful procedure was reported in $10 \%$ of cases. Minor dacryocystitis appeared in $18.9 \%$ of cases, while complete resolution of epiphora was confirmed in $77 \%$ of cases (23\% of cases showed grade I epiphora). Patency of stents: median patency 490 days (15 months), range 11 to 730 days; 1 year after stent placement patency was 0.51 and long term patency rate for 2 years was 0.31 . Opinion survey of the 68 patients: satisfaction with the technique, the procedure and prosthesis placement was $41 \%$; satisfaction whilst the stent remained patent was $60.8 \%$.
\end{abstract}


Encuesta de satisfacción a los 68 pacientes: con respecto a la técnica, procedimiento y prótesis estaban satisfechos un $41 \%$. Mientras la prótesis se mantuvo permeable se encontraban satisfechos un $60,8 \%$. Conclusión: La prótesis Tear-Leader es de fácil colocación, con escasas molestias para el paciente. Presenta una permeabilidad similar al resto de stents del mercado.

Palabras clave: Glándula y conducto lacrimal, radiología intervencionista, dacriocistitis crónica, stent orbitario lacrimal, prótesis recta de Wacrees.
Conclusion: Tearleader stent placement is easy to perform and comfortable for patients. TearLeader placement gave a patency outcome similar to other stents (Arch Soc Esp Oftalmol 2009; 84: 515-522).

Key words: Lacrimal gland and duct, interventional procedures, chronic dacryocystitis, lacrimal orbital stent, Wacrees upright protheses.

\section{INTRODUCCIÓN}

La dacriocistitis es consecuencia de infecciones e inflamación crónica de la vía lacrimal. Esta situación va desarrollando una obstrucción del conducto lacrimal y produciendo epífora. Este síntoma es un problema oftalmológico frecuente que representa el 3\% de las visitas clínicas, se suele acompañar secreción e hiperemia conjuntival que condicionan molestias para el paciente (1).

En los últimos años se han desarrollado las prótesis nasolacrimales que se colocan bajo control fluoroscópico y que se han descrito como un tratamiento alternativo a la cirugía para la obstrucción del conducto lacrimal (2-4).

El objetivo de este trabajo es estudiar el stent de Tear-Leader (InterV/PBN Medicals, Denmark) en el tratamiento de la obstrucción del conducto lacrimal, evaluando los resultados clínicos y el grado de satisfacción de los pacientes así como realizar el estudio de la permeabilidad del stent.

\section{SUJETOS, MATERIAL Y MÉTODO}

Durante dos años, se han colocado 74 stents nasolacrimales Tear-Leader (PBN) para el tratamiento de la epífora por obstrucción del conducto nasolacrimal en nuestro Servicio de Radiología (Sección de Radiología Intervencionista).

El Servicio de Oftalmología remitía a los pacientes después de una valoración clínica donde se realizaba una exploración inicial de la epífora. Según la clasificación de Munk la epífora se gradaba del 0 al 4 (según el número de veces que tiene que limpiarse el ojo al día). Grado 0: no epífora, Grado 1: menos de 2 veces al día, Grado 2: de 2 a 4 veces al día, Grado 3: de 4 a 10 veces al día, Grado 4: más de 10 veces al día.

A la llegada al Servicio de Radiología, se les realizaba en primer lugar una dacriocistografía para valorar el nivel de la obstrucción. Después del estudio radiológico, se les explicaba en qué consistía el procedimiento dándoles el consentimiento informado.

El control de imagen se realizaba en una sala de Angiografía digital Allura Xper FD 20 (Philips, Holanda) con estación 3DRa y view forum.

Los criterios de inclusión para la colocación del stent fueron: la obstrucción completa del conducto lacrimal con presencia de saco lacrimal, permeabilidad de los canalículos lacrimales y de los puntos lacrimales, así como la ausencia de infección aguda.

Se han excluído del trabajo aquellos pacientes que abandonaron el estudio y no se sometieron a los controles evolutivos.

Los stents nasolacrimales se colocaban por radiólogos vasculares intervencionistas con importante experiencia en la especialidad y habituados con el manejo de stents. Tras la colocación del stent se realizaban controles periódicos evolutivos mensuales durante tres meses citando al paciente y realizando lavados con suero fisiológico del conducto lacrimal. Si existía alguna incidencia o el paciente refería la recidiva de la epífora, se realizaba una nueva dacriocistografía para la valoración exacta de la permeabilidad de la prótesis. A partir de los tres meses el contacto con los pacientes era trimestral de forma telefónica. Si en el intervalo existía alguna incidencia se ponían en contacto con nosotros y les realizábamos una nueva exploración radiológica y en el caso de que existiera obstrucción del stent, éste era retirado por el servicio de Otorrinolaringo- 
TABLA I. Recogida de datos del stent lacrimal

\section{Nombre:}

Edad: . años

NHC:

Fecha de la colocación del stent: dd/mm/aa.

Fecha de recidiva de los síntomas: dd/mm/aa (aprox).

Durante el tiempo de funcionamiento del stent:

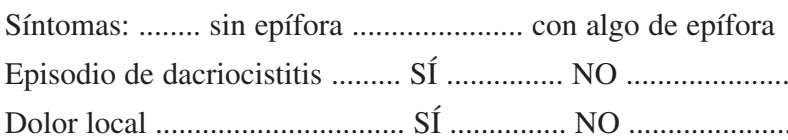

Grado de satisfacción con funcionamiento de stent:
1. muy satisfecho
2. bastante satisfecho
3. poco satisfecho
4. nada satisfecho

Grado satisfacción en general:

1. muy satisfecho

2. bastante satisfecho

3. poco satisfecho

4. nada satisfecho

logía. A partir de los tres meses de colocación del stent, a todos los pacientes se les realizó una encuesta telefónica para conocer el grado de satisfacción (en una escala de 1 al 4), preguntando si estuvieron satisfechos mientras el stent funcionaba, y en relación al procedimiento en general, incluyendo tanto el momento de la colocación, el trato recibido por el personal, así como la mejoría clínica de la epífora y sus complicaciones posteriores.

Si en el intervalo existía alguna incidencia o ante la sospecha de malfuncionamiento de la prótesis, los pacientes se ponían en contacto con nosotros y les realizábamos una nueva exploración clínicoradiológica que incluía la evolución de la epífora y la aparición de alguna incidencia como episodios de dacriocistitis aguda o dolor local (tabla I).

\section{Descripción del stent}

El set de Tear-Leader (TearLeader Stent, interV/PBN Medical, Denmark) consta de un stent de diámetro de $6 \mathrm{~F}$ y $35 \mathrm{~mm}$ de longitud, un dilatador, un empujador para la colocación de la endopró-

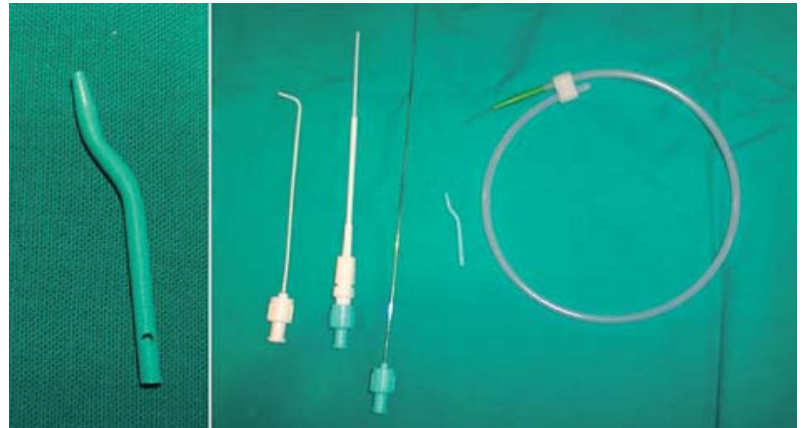

Fig. 1: Componentes del set de prótesis nasolacrimal de Tear Leader.

tesis, una guía atraumática de nitinol con $7 \mathrm{~cm}$ de punta flexible e hidrofílica y un catéter para la dacriocistografía. El stent presenta una configuración en $\mathrm{S}$ afilada por el extremo más proximal (fig. 1).

El procedimiento se realizó de forma ambulatoria en todo los casos, con anestesia local periorbitaria (lidocaína al 1\%) y sedación en caso necesario. Para la descongestión de la mucosa nasal se introdujo una gasa empapada en anestésico local y adrenalina. Los pacientes recibieron amoxicilina oral $500 \mathrm{mg} / 8$ horas, 24 horas antes del procedimiento y los 6 días posteriores. En el momento de la manipulación se administraron antibióticos y esteroides tópicos en gotas. En todos los casos tras la colocación del stent se realizaron lavados del conducto lacrimal con suero fisiológico.

El estudio ha sido prospectivo con estadística descriptiva y se ha estudiado la permeabilidad del stent nasolacrimal usando las curvas de supervivencia de Kaplan Meier.

\section{RESULTADOS}

Todos los pacientes del estudio presentaban un grado IV de epífora con obstrucción completa del conducto lacrimal. No existía ningún paciente con epífora de origen traumático. El rango de edad de los pacientes fue de 18-84 años con una media de edad de 61,8 años, 22 hombres y 46 mujeres. Hemos incluido en el trabajo únicamente los pacientes en los que la colocación del stent fue exitosa.

El tiempo de fluoroscopia necesario durante la manipulación fue de 59 segundos (rango 40 segundos a 1,5 minutos).

Durante la colocación del stent no existieron complicaciones mayores. El momento de la implan- 


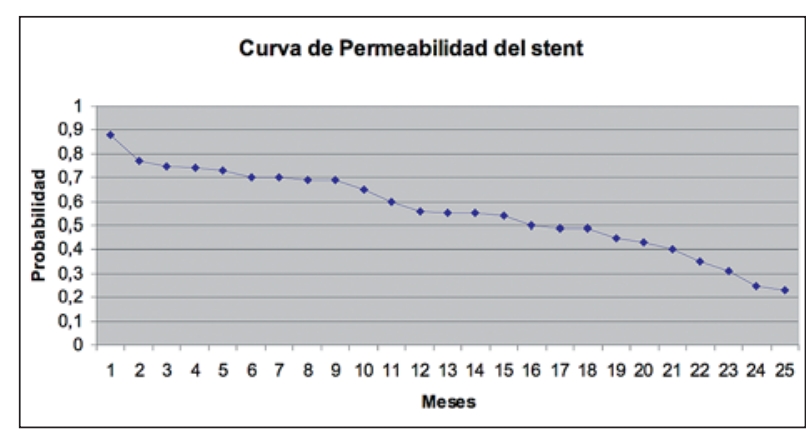

Fig. 2: Curva actuarial de Kaplan Meier. Expresa la probabilidad de permeabilidad del stent nasolacrimal en los diferentes meses que ha durado el seguimiento hasta un total de 2 años.

tación de la prótesis los pacientes la refieren como dolorosa en el $10 \%$ de los casos, cabe destacar que un $81 \%$ de ellos describieron el momento de la colocación de la prótesis como «nada molesto». Sólo 3 pacientes presentaron epistaxis leve durante la manipulación, que cedió espontáneamente al finalizar la colocación y realizar lavados con suero fisiológico del stent.

\section{Seguimiento clínico}

(Exploración clínica mensual durante tres meses y consulta telefónica trimestral posterior, si aparecía incidencia: realización de dacriocistografía).

Durante el tiempo en el que los stents estuvieron permeables la mejoría en cuanto al lagrimeo fue notable aunque persistió epífora grado I en un 23\% de los pacientes. Tras la colocación del stent y con éste funcionante, persistieron episodios aislados de dacriocistitis aguda en el 18\% de los casos implantados que se resolvieron con éxito tras la administración de antibioticoterapia tópica, aunque en cuatro pacientes fue el inicio de obstrucción de stent posterior. En cinco casos existió salida inadvertida del stent $(6,1 \%)$ que se constató al relizar una dacriocistografía de control.

En total se reevaluaron por sospecha clínica de obstrucción 31/68 pacientes, en los que en 15 de ellos de demostró la obstrucción del stent.

\section{Permeabilidad del stent}

El estudio de la permeabilidad del stent mostró una mediana de 490 días (15 meses), con un rango
Tabla II. Análisis de permeabilidad. Estadística descriptiva

\begin{tabular}{lccc}
\hline Días & Máxima & Media & Mediana \\
\hline Tear-Leader stent & 731 & 267,9 & 490 \\
& & $\begin{array}{c}\text { desviación típica } \\
214,1\end{array}$ \\
\hline \hline
\end{tabular}

de 11 a 730 días, al año el $51 \%$ se encontraban permeables y a 2 años el $31 \%$. La gráfica de la permeabilidad viene representada por la curva actuarial de Kaplan Meier, se ha expresado en meses para ser más comprensible (fig. 2).

\section{Encuesta de satisfacción:}

Mientras la prótesis fue funcionante y mejoraron ostensiblemente los síntomas, los pacientes se encontraban satisfechos con el stent un 60,8\% (incluimos pacientes satisfechos y muy satisfechos). Si a los pacientes les preguntábamos en conjunto sobre: el procedimiento, que incluía la técnica, el trato con el personal sanitario y también le preguntábamos sobre la mejoría clínica tras la colocación de la prótesis incluyendo la obstrucción del stent se encontraban satisfechos sólo un $41 \%$ de los pacientes consultados.

\section{DISCUSIÓN}

La epífora es uno de los síntomas más frecuentes de los pacientes que acuden a una consulta de oftalmología. Este problema puede deberse a múltiples causas que se pueden agrupar en dos grandes categorías: las alteraciones de tipo irritativo, que producen un lagrimeo reflejo y el déficit funcional u orgánico de drenaje, siendo la obstrucción adquirida del conducto lacrimal la más frecuente. Esta enfermedad suele presentarse en forma de conjuntivitis de repetición, dermatitis irritativa de los párpados, secreción crónica, formación de dacrioceles e incluso dacriocistitis aguda a veces de forma recidivante. Habitualmente es unilateral, pero puede aparecer de forma bilateral y afecta a aproximadamente el $2 \%$ de la población. Es mucho más frecuente en individuos de edad avanzada aunque puede aparecer a cualquier edad.

El tratamiento tradicional es la dacriocistorrinostomía por vía externa. Esta técnica en manos exper- 
tas tiene un éxito del 90\%, actualmente es la técnica de rescate de todas las demás (4). En los últimos años han ido apareciendo nuevas técnicas como la dacriocistorrinostomía endoscópica con láser. Esta intervención presenta la ventaja de no precisar incisión externa y existe un menor riesgo de sangrado, sin embargo presenta un porcentaje de fracaso del 17 al $30 \%$ de los casos (5).

En 1995 se publica un nuevo tratamiento como alternativa a la dacriocistorrinostomía, Song et al (6) describen un nuevo método de colocación retrógrada de una prótesis de poliuretano. Los primeros resultados fueron prometedores con un porcentaje de permeabilidad del 85 al 98\% (7-9), pero el seguimiento de estos pacientes estudiados era de menos de un año. Muchos radiólogos intervencionistas comenzaron a usar este stent ya que ofrecía seguridad, era relativamente sencillo de implantar y permitía manejos quirúrgicos posteriores si la prótesis se obstruía. Pero los resultados a medio y largo plazo no fueron tan prometedores, incluso Song recomendó no usar el stent como primera opción en el tratamiento de la obstrucción del conducto nasolacrimal (10). Un trabajo de Yazini et al llegaba a la misma conclusión (11), la permeabilidad iba disminuyendo ostensiblemente con el tiempo, aportando cifras de éxito del $69 \%$. Además este estudio no incluía pacientes en los que la obstrucción del conducto lacrimal era de causa traumática, ya que en estos casos se ha demostrado que la tasa de fracasos en el funcionamiento del stent es mayor $(6,12)$. Otros autores llegaban a conclusiones similares aportando cifras de permeabilidad del $56 \%$ a los 2 años de seguimiento (13). Otro inconveniente del stent de Song era que se requería sacos lacrimales grandes para conseguir la adecuada expansión del extremo proximal de la prótesis (11). Existen estudios que muestran mejores porcentajes de permeabilidad del stent, como el de Lanciego et al, donde a los 15 meses después de su colocación el 85,8\% de las mismas estaban funcionantes (16).

La causa principal del fallo del stent se demostró que era debido a la inflamación crónica que se producía alrededor de la prótesis y generaba tejido de granulación (14). Además, la morfología del saco lacrimal empeoraba tras la retirada de un stent obstruido ya que éste se contraía y disminuía su tamaño, dificultando la colocación de una nueva prótesis (11).

Nosotros comenzamos a usar el stent de Song porque era el único disponible en el mercado (Ferrer-Puchol M, Esteban-Hernández E, Jornet-
Fayos J. Obstruction of the lacrimal system. Treatment and long term results with polyurethane Song stent versus TearLeader-stent. In: Annual Meeting and Postgraduate Course of the Cardiovascular and Interventional Radiological Society of Europe, 2005 Abstract Book poster 30, p 164). El principal inconveniente que encontramos era que su colocación resultaba dolorosa y molesta para el paciente (sobretodo el momento donde se introducía el gancho por las fosas nasales para capturar la guía). Nos llama la atención que en la literatura no se haga hincapié en este hecho. También observamos casos de sangrado nasal transitorio, que sí lo describen otros autores, incluso con algún caso de epistaxis grave (17).

Un hecho a tener en cuenta es que con el stent de Song (al ser el más ampliamente implantado) existen estudios de permeabilidad a más largo plazo, se conoce que a los 5 años de colocación de la prótesis únicamente están permeables el 19,2\% de los casos (18).

Todos estos hechos han contribuido a que aparecieran, o bien modificaciones al stent del Song, como el que describen Lanciego et al, donde sus resultados con el nuevo diseño mejora la permeabilidad (19), o diseños nuevos como el TearLeader (20) que no presenta la porción abalonada en el extremo proximal además de tener una morfología en «s» itálica. Para nuestro criterio, la ventaja fundamental del nuevo stent es que no precisa de gancho para extraer la guía por los orificios nasales, ya que dispone de un catéter que se puede dirigir una vez atravesada la obstrucción hacia la parte anterior y al introducir la guía, ésta sale espontáneamente por los orificios nasales.

Nuestro grupo comenzó con este nuevo diseño de stent, que representó inmediatamente mayor facilidad en el manejo y que se convirtió en un procedimiento prácticamente indoloro para el paciente (sólo el 10\% de los pacientes encuestados referían el procedimiento como molesto). Además disminuyó ostensiblemente el tiempo empleado en su colocación, con la ventaja que supone un tiempo mínimo de fluoroscopia.

Hay que destacar como resultado negativo, que mientras el stent TearLeader estuvo funcionante persistía epífora, aunque mínima (grado I), en un $23 \%$ de los pacientes y se presentaron casos de dacriocistitis (18\%). Estos resultados los atribuimos a que la porción proximal del stent (la que descansa en el saco lacrimal) es demasiado estrecha y favorece, en algunas situaciones, que exista un dre- 
naje pobre de la lágrima a través de la prótesis. Otro inconveniente que encontramos en el diseño de este nuevo stent es, que existe la posibilidad de salida inadvertida del mismo, como referimos en los resultados de nuestro estudio, donde en cinco pacientes que acudieron para control por presentar aumento de la epífora se descubrió que el stent no se encontraba en el conducto lacrimal.

En cuanto a la permeabilidad del stent nuestros datos son superponibles a los datos publicados en la literatura, con la limitación de que nuestro estudio dura sólo dos años, desconociendo qué permeabilidad tendremos a largo plazo. Existen escasos estudios con este nuevo diseño de stent. Wilhelm et al (20) evalúan el stent TearLeader comparado con el stent de Song, siendo llamativo que la permeabilidad es mejor en los stents de TearLeader y que además el revestimiento hidrofílico del stent impide que existan reacciones granulomatosas como respuesta a cuerpo extraño, señalándose este hecho como papel fundamental en la permeabilidad de la prótesis, pero la limitación de este estudio es que está realizado en conejos y que el seguimiento del estudio es únicamente de 3 meses.

Creemos que el stent es relativamente seguro con aparición de pocas complicaciones y de fácil manejo, aunque también queremos resaltar una complicación importante ocurrida recientemente con este stent y descrita por Lanciego et al (21), donde tras la implantación de la prótesis la paciente comenzó con un intenso edema por celulitis y pérdida de visión. En el fondo de ojo realizado se objetivó atrofia y palidez en el disco óptico; un mes después se le practicó dacriocistorrinostomía que se efectuó sin complicaciones. La pérdida de visión de la paciente se atribuyó a la compresión de la raíz nerviosa por la celulitis orbitaria que se extendió desde el suelo de la órbita hasta el músculo recto interno causando una isquemia irreversible en el nervio óptico. Sin embargo, aparte de esta complicación, no tenemos noticias de que se hayan descrito en la literatura otras complicaciones similares y pensamos que en principio es un procedimiento con escasos riesgos, pero creemos importante hacer hincapié en la administración de antibioticoterapia profiláctica para prevenir posibles infecciones.

La encuesta realizada a los pacientes mostraba un grado de satisfacción del 60,8\% mientras el stent funcionó, y la opinión de los pacientes al preguntarles por todo el procedimiento en general fue baja (tan solo del 41\%). Sabemos que estos resultados tienen importantes limitaciones que los sesgan, ya que las contestaciones de los pacientes a la encuesta están sometidas a múltiples influencias (estado de ánimo, concomitancia con otras patologías, etc). Aún así, creemos que los pacientes tenían unas expectativas mucho mejores que los resultados obtenidos.

En resumen creemos que las prótesis nasolacrimales ofrecen una alternativa terapéutica interesante en casos de obstrucción del conducto lacrimal. La ventaja principal de la prótesis de TearLeader se encuentra en que su implantación es sencilla y poco molesta para el paciente. Aún así, pensamos que los diseños se tienen que mejorar para conseguir permeabilidades mayores, precisándose más estudios con mayor tiempo de seguimiento de los pacientes. Quizás el futuro esté en prótesis sencillas de colocar y de permanencia limitada, ya que parece que la evolución del stent es hacia la obstrucción en la mayoría de los casos.

\section{BIBLIOGRAFÍA}

1. Linberg JV, McCormick SA. Primary acquired nasolacrimal duct obstruction. A clinicopathologic report and biopsy technique. Ophtalmology 1986; 93: 1055-1063.

2. Song HY, Yin YH, Kim JH, Suh SW, Yoon HK, Kang SG, et al. Non-surgical placement of a nasolacrimal polyurethane stent: long-term effectiveness. Radiology 1996; 200: 759-763.

3. Munk PL, Lin DT, Morris DC. Epiphora: treatment by means of dacryocystoplasty with balloon dilation of the nasolacrimal drainage apparatus. Radiology 1990; 177: 687-690.

4. Blanco Mateos G, Santos Pérez J. Dacriocistorrinostomía endonasal: nueva perspectiva en el tratamiento de la obstrucción del conducto nasolacrimal. Arch Soc Esp Oftalmol 2004; 79: 99-101.

5. Ben Simon GJ, Joseph J, Lee S, Schwarcz RM, McCann JD, Goldberg RA. External versus endoscopic dacryocystorhrinostomy for acquired nasolacrimal duct obstruction in a tertiary referral center. Ophthalmology 2005; 112: 1463-1468.

6. Song HY, Jin YH, Kim JH, Huh SJ, Kim YH, Kim TH, et al. Nonsurgical placement of a nasolacrimal polyurethane stent. Radiology 1995; 194: 233-237.

7. Wilhelm KE, Hofer U, Textor HJ, Böker T, Struk H, Schild HH. Nonsurgical fluoroscopically guided dacryocystoplasty of common canalicular obstructions. Cardiovasc Intervent Radiol 2000; 23: 1-8.

8. Pulido-Duque JM, Reyes R, Carreira JM, Vega F, Górriz E, Pardo MD, et al. Treatment of complete and partial obstruction of the nasolacrimal system with polyurethane stents: Initial experience. Cardiovasc Intervent Radiol 1998; 21: 41-45.

9. Pinto IT, Paúl L, Grande C. Nasolacrimal polyurethane stent: complications with CT correlation. Cardiovasc Intervent Radiol 1998; 21: 450-453. 
10. Ko GY, Song HY, Seo TS, Kim TH, Sung KB, Yoon HK. Obstruction of the lacrimal system: treatment with a Covered, Retrievable, Expandable Nitinol Stent versus a Lacrimal Polyurethane stent. Radiology 2003; 227: 270-276.

11. Yazici Z, Yazici B, Parlak M, Tuncel E, Ertürk H. Treatment of nasolacrimal duct obstruction with polyurethane stent palcement: long-term results. AJR Am J Roentgenol 2002; 179: 491-494.

12. Yazici B, Yazici Z, Parlak M. Treatment of nasolacrimal duct obstruction in adults with polyurethane stent. Am J Ophthalmol 2001; 131: 37-43.

13. Pinilla I, Fernández-Prieto AF, Asencio M, Arbizu A, Peláez N, Frutos R. Nasolacrimal stents for the treatment of epiphora: technical problems and long-term results. Orbit 2006; 25: 75-81.

14. Song HY, Lee DH, Ahn H, Kim JH, Kang SG, Yoon HK, et al. Lacrimal system obstruction treated with lacrimal polyurethane stents: outcome of removal of occluded stents. Radiology 1998; 208: 689-694.

15. Kim JH, Shin JH, Song HY, Kang W, Lim JO, Park SH, et al. Long-term results and factor affecting patency after removal of nasolacrimal stents. J Vasc Interv Radiol 2006; 17: 1125-1130.
16. Lanciego C, De Miguel S, Perea M, Cano C, García IR, Dávila J, et al. Nasolacrimal stents in the management of epiphora: medium-term results of a multicenter prospective study. J Vasc Interv Radiol 2001; 12: 701-710.

17. Pabón IP, Diaz LP, Grande C, de la Cal López MA. Nasolacrimal polyurethane stent placement for epiphora: technical long-term results. J Vasc Interv Radiol 2001; 12: 6771.

18. Song HY, Lee DH, Ahn H, Seo TS, Ko GY. Intervention in the lacrimal drainage system. Cardiovasc Intervent Radiol 2002; 25: 165-170.

19. Lanciego C, De Miguel S, Padilla M, Perea M, RodriguezMerlo R, García-García L. Nasolacrimal stenting: toward improving outcomes with a simple modification of the song stent. Cardiovasc Intervent Radiol 2006; 29: 586-594.

20. Wilhelm KE, Grabolle B, Urbach H, Tolba R, Schild H, Paulsen F. Evaluation of polyurethane nasolacrimal duct stent: in vivo studies in New Zealand Rabbits. Cardiovasc Interv Radiol 2006; 29: 846-853.

21. Lanciego C, Bracamonte A, Méndez-Cendón JC, César Gómez, Padilla M, García-García L. Loss of vision as a complication of nasolacrimal stent placement. J Vasc Interv Radiol 2004; 15: 1027-1028. 